\title{
Coronary stents with inducible VEGF/HGF- secreting UCB-MSCs reduced restenosis and increased re-endothelialization in a swine model
}

Hyun-Kyung Chang ${ }^{1}$, Pyung-Hwan Kim ${ }^{1,2}$, Dong Wook Kim¹, Hyun-Min Cho ${ }^{1}$, Mi Jin Jeong ${ }^{3}$, Dea Han Kim4, Yoon Ki Joung ${ }^{3}$, Kyung Seob Lim ${ }^{5,6}$, Han Byul Kim ${ }^{5}$, Han Cheol Lim ${ }^{5}$, Dong Keun Han ${ }^{4}$, Young Joon Hong ${ }^{5}$ and Je-Yoel Cho ${ }^{1}$

\begin{abstract}
Atherosclerotic plaques within the vasculature may eventually lead to heart failure. Currently, cardiac stenting is the most effective and least invasive approach to treat this disease. However, in-stent restenosis is a complex chronic side effect of stenting treatment. This study used coronary stents coated with stem cells secreting angiogenic growth factors via an inducible genome-editing system to reduce stent restenosis and induce re-endothelialization within the artery. The characteristics of the cells and their adhesion properties on the stents were confirmed, and the stents were transplanted into a swine model to evaluate restenosis and the potential therapeutic use of stents with stem cells. Restenosis was evaluated using optical coherence tomography (OCT), microcomputed tomography $(\mathrm{mCT})$ and angiography, and re-endothelialization was evaluated by immunostaining after cardiac stent treatment. Compared to a bare metal stent (BMS) or a parental umbilical cord blood-derived mesenchymal stem cell (UCB-MSC)-coated stent, the stents with stem cells capable of the controlled release of hepatocyte growth factor (HGF) and vascular endothelial growth factor (VEGF) successfully reduced restenosis within the stent and induced natural re-endothelialization. Furthermore, UCB-MSCs exhibited the ability to differentiate into endothelial cells in Matrigel, and HGF and VEGF improved this differentiation. Our study indicates that stents coated with UCB-MSCs secreting VEGF/HGF reduce the restenosis side effects of cardiac stenting with improved re-endothelialization.
\end{abstract}

\section{Introduction}

Coronary artery disease is an angiocardiopathy that severely impairs health, and it remains the principal cause of mortality worldwide. The goal of treatment is the restoration of blood flow in the clogged artery to a nearnormal rate ${ }^{1-3}$. Coronary stents are a widely used treatment strategy to keep the arteries open. However,

\footnotetext{
Correspondence: Je-Yoel Cho (jeycho@snu.ac.kr)

'Department of Biochemistry, BK21 PLUS Program for Creative Veterinary Science Research and Research Institute for Veterinary Science, College of Veterinary Medicine, Seoul National University, Seoul, South Korea

${ }^{2}$ Department of Biomedical Laboratory Science, College of Medical Science, Konyang University, Daejeon, South Korea
}

Full list of author information is available at the end of the article. restenosis and stent thrombosis limit the success of stent treatment. Delayed or incomplete endothelial regeneration is a key factor of these events.

The endothelialization of coronary stents decreases instent restenosis ${ }^{4-7}$. This process is an important factor in thrombosis prevention and the reduction of vascular smooth muscle cells (VSMCs) proliferation and migration. Therefore, a coronary stent that is capable of rapid surface endothelialization may become a next-generation stent $^{7-10}$. We used a very effective combination strategy of gene and cell therapies, in which genome-edited stem cells released proangiogenic growth factors, to improve re-endothelialization.

\section{(c) The Author(s) 2018}

(c) (i) Open Access This article is licensed under a Creative Commons Attribution 4.0 International License, which permits use, sharing, adaptation, distribution and reproduction in any medium or format, as long as you give appropriate credit to the original author(s) and the source, provide a link to the Creative Commons license, and indicate if changes were made. The images or other third party material in this article are included in the article's Creative Commons license, unless indicated otherwise in a credit line to the material. If material is not included in the article's Creative Commons license and your intended use is not permitted by statutory regulation or exceeds the permitted use, you will need to obtain permission directly from the copyright holder. To view a copy of this license, visit http://creativecommons.org/licenses/by/4.0/. 
Vascular endothelial growth factor (VEGF) is one of the most effective signaling proteins that stimulates vasculogenesis $^{11,12}$. Hepatocyte growth factor (HGF) is a pleiotrophic factor that induces motogenesis, mitogenesis, survival, and morphogenesis in some cell types ${ }^{13-15}$. Therefore, the integration of these genes into the genome of human umbilical cord blood-derived mesenchymal stem cells (U-Ms) enhances the ability of these cells to stimulate angiogenesis. We used the TALEN genomeediting system to integrate these genes into stem cells and introduce targeted double-strand breaks into the chromosome 19 safe-harbor site. We controlled gene expression with doxycycline using the Tet-on system. Our previous studies demonstrated that VEGF and HGF-secreting U-Ms (VEGF/U-Ms and HGF/U-Ms) enhanced angiogenesis in a rat myocardial infarction model and mouse hind limb ischemia model ${ }^{13,16}$. VEGF/ U-Ms and HGF/U-Ms were very effective and powerful cell therapy systems for the restoration of blood vessels and blood flow.

Stents coated with polydopamine (pDA), fibronectin (FN), and extracellular matrix (ECM) enhance stem cell adhesion, including MSCs, to metallic stents ${ }^{17}$. An ECM is a biocompatible and cell-supporting substance that provides cells with mechanical and physiological support to increase cell survival, adhesion, proliferation, and differentiation $^{18,19}$. The ECM also traps and holds some growth factors and soluble molecules via proteoglycans, which are its primary components ${ }^{20}$. However, the ECM requires a strong connector to attach to metal surfaces, and pDA and FN are used as chemical connectors. FN recognizes and binds to ECM molecules via integrin, and its carboxyl termini covalently bind to pDA. Therefore, the FN-pDA layers serve as linkers to immobilize ECM molecules, such as fibrin, collagen, heparin, and fibronectin, on the surface $^{21}$. Dopamine is a strong adhesive molecule derived from the muscle ${ }^{22}$. Dopamine binds firmly to organic and inorganic surfaces via a catechol that consists of a benzene ring with two hydroxyl groups. pDA also provides a functional amine group to immobilize molecules on the surface. Stem cells may be efficiently seeded after coating the stents with these three components.

This study investigated the potential of stents seeded with angiogenic growth factor-secreting MSCs to enhance re-endothelialization and reduce restenosis via rapid re-endothelialization. We loaded coronary stents with functional stem cells (VEGF/U-Ms and HGF/U-Ms) and assessed the efficacies of in-stent stenosis reduction and coronary artery re-endothelialization in a swine model.

\section{Materials and methods}

\section{Cell culture and cell preparation}

UCB-MSCs (U-Ms) isolated from human umbilical cord blood (hUCB) were kindly provided by the Kang laboratory at Seoul National University. Cells were isolated from hUCB as previously described ${ }^{23}$. The Borame Institutional Review Board and Seoul National University approved the U-M isolation procedure (IRB No. 0603/ 001-002-07C1). The U-Ms were maintained in mesenchymal stem cell medium (KSB-3, Kangstem Biotech, South Korea) supplemented with KSB-3 and 10\% fetal bovine serum (Rocky Mountain Biologicals Inc., MT, USA) at $37^{\circ} \mathrm{C}$ in $5 \% \mathrm{CO}_{2}$. Cells were transfected with NEON using the TALEN system and HGF- or VEGFsecreting plasmids as previously described ${ }^{13,16}$.

\section{Viability assay}

Live cells on precoated stent material were imaged by fluorescence microscopy after labeling with green fluorescent dye (PKH67, Sigma, USA). The metal materials were coated with polydopamine, fibronectin, and ECM. Cell viability was tested using the crystal violet assay and cell counting. Cells $\left(5 \times 10^{4}\right)$ were seeded onto stent material coated with fibronectin and an extracellular matrix layer and incubated for 7 days. A crystal violet solution $(50 \mu \mathrm{l}$ of $0.5 \%)$ was added, and an image of the stained cells was captured. Methanol $(200 \mu \mathrm{l})$ was added to each well and incubated for $20 \mathrm{~min}$ at RT. The cell density was detected at $570 \mathrm{~nm}$ using a spectrophotometer (Epoch, BioTek, VT, USA). Cells were counted using a hemocytometer after trypan blue (Gibco, NY, USA) staining.

\section{Western blotting (WB)}

WB was performed to confirm growth factor expression, as previously described ${ }^{13,16}$. Transfected cells $\left(5 \times 10^{5}\right)$ were seeded onto the stent material for $24 \mathrm{~h}$ and treated with Dox $(5 \mu \mathrm{g} / \mathrm{ml})$ for 2 days. The conditioned medium was collected and precipitated using trichloroacetic acid (TCA, Sigma, USA). The pellet was dissolved in $200 \mu \mathrm{l}$ of RIPA buffer (Thermo, IL, USA). SDS-PAGE was performed using a $1 \%$ acrylamide gel. HGF (R\&D Systems, MN, USA) and VEGF (Cell Signaling, MA, USA) antibodies were used at a 1:1000 dilution.

\section{Conventional PCR and real-time quantitative PCR}

Conventional PCR and real-time PCR were performed 7 days after cell seeding on the stent material to assess MSC marker expression. Supplementary Table 1 lists the primer sequences targeting CD166, CD105, CD90, CD45, CD14, and GAPDH. PCR was performed using the GoTaq polymerase (Promega, MN, USA) according to the following protocol: one cycle of $95^{\circ} \mathrm{C}$ for $5 \mathrm{~min}$, 35 cycles of $95^{\circ} \mathrm{C}$ for $30 \mathrm{~s}, 60.1^{\circ} \mathrm{C}$ for $30 \mathrm{~s}, 72^{\circ} \mathrm{C}$ for $30 \mathrm{~s}$, and a final cycle of $72{ }^{\circ} \mathrm{C}$ for $3 \mathrm{~min}$. PCR products were evaluated using a $1.5 \%$ agarose gel. Real-time PCR was performed using the same PCR conditions and a SYBR Green-based method. 


\section{Tube formation}

Cells $\left(5 \times 10^{4}\right.$ cells/well) were seeded onto a layer of BD Matrigel (BD Biosciences, CA, USA) in 24-well plates and exposed to high-glucose DMEM (HyClone, UT, USA) containing $2 \%$ FBS for $48 \mathrm{~h}$. MSCs alone were used as a control. Cells were incubated for $12 \mathrm{~h}$ to allow the formation of tube-like structures. Tube formation was analyzed via counting the number of branches per highpower field.

\section{Stent preparation}

The coating protocol and its effect on enhancing cell adhesion were published previously ${ }^{17}$. Briefly, an $18 \mathrm{~mm} \times 1.8 \mathrm{~mm}$ cobalt-chromium stent was incubated with $1 \mathrm{mg} / \mathrm{ml}$ of dopamine (Sigma, BT, USA) dissolved in $10 \mathrm{mM}$ Tris buffer ( $\mathrm{pH}$ 8.5). The pDA-coated CoCr stent was washed three times using ultrasonication for $10 \mathrm{~min}$. The pDA-coated $\mathrm{CoCr}$ stent was washed with deionized water and dried in air. The pDA-coated $\mathrm{CoCr}$ stent was placed in a $50-\mu \mathrm{g} / \mathrm{ml} \mathrm{FN} \mathrm{solution} \mathrm{at} 37{ }^{\circ} \mathrm{C}$ overnight and washed for $10 \mathrm{~min}$. NIH3T3 fibroblasts (ATCC, VA, USA), at a density $1 \times 10^{4}$ cells $/ \mathrm{cm}^{2}$, were cultured on the pDA-FN-coated CoCr stent for 10 days to form the ECM layer. Cells were decellularized using $20 \mathrm{mM} \mathrm{NH}_{4} \mathrm{OH}$ and $0.5 \%$ Triton X-100. The decellularized surface was washed with PBS and treated with $50 \mu \mathrm{g} / \mathrm{ml}$ of RNase A and 50 units $/ \mathrm{ml}$ of DNase I for $24 \mathrm{~h}$ at $37^{\circ} \mathrm{C}$. HGF or VEGF/UMs $\left(2.4 \times 10^{6}\right.$ cells $)$ were seeded on the pDA-FN-ECMcoated stent.

\section{Confirmation of cell adhesion}

The stents were coated with fibronectin and extracellular matrix, as previously described ${ }^{10}$. Cells $\left(2.4 \times 10^{6}\right)$ were seeded on the stents and incubated for $12 \mathrm{~h}$ in a $\mathrm{CO}_{2}$ incubator. Cells on the stent were detected using fluorescence microscopy (July, NanoEntek, South Korea). Scanning electron microscopy (SEM) analysis was performed to assess the cells remaining on the stent after transplantation. The samples were rinsed with $2.5 \%$ glutaraldehyde in $\alpha$-MEM without serum and fixed for $30 \mathrm{~min}$ at RT. The samples were fixed in $2.5 \%$ glutaraldehyde in $0.1 \mathrm{M} \mathrm{Na-cacodylate} \mathrm{(pH} \mathrm{7.2)} \mathrm{with} 0.1 \mathrm{M}$ sucrose for an additional $30 \mathrm{~min}$ at RT. The samples were treated with $1 \%$ osmium tetroxide in distilled water for 1 $\mathrm{h}$, followed by dehydration through a graded series of ethanol solutions from $70 \%, 80 \%, 95 \%$ to $100 \%$. A freezedryer was used to dry the samples. Samples were mounted on aluminum holders and coated with a 10-nm conducting layer of gold platinum. Samples were examined in an SEM (Jeol JSM7400F, Tokyo, Japan) using a voltage of $10 \mathrm{kV}$.

\section{Transplantation into the swine model}

The Ethics Committee of Chonnam National University Medical School and Chonnam National University
Hospital approved this animal study (CNU IACUCH-2013-12), which conformed to the Guide for the Care and Use of Laboratory Animals published by the US National Institutes of Health (NIH Publication No. 85-23, revised 1996). Yorkshire $\times$ Landrace F1 crossbred castrated male pigs $(20-25 \mathrm{~kg})$ were observed in the laboratory animal center of Chonnam National University Medical Institute for 5-10 days prior to the experiment.

Pigs were anesthetized with zolazepam and tiletamine (2.5 mg/kg, Zoletil50 ${ }^{\circ}$, Virvac, Caros, France), xylazine (3 mg/kg, Rompun ${ }^{\oplus}$, Bayer AG, Leverkusen, Germany), and azaperone $\left(6 \mathrm{mg} / \mathrm{kg}\right.$, Stresnil ${ }^{\circ}$, Janssen-Cilag, Neuss, Germany). An intravenous (IV) catheter was placed in the marginal ear vein for the administration of fluids and emergency drugs, such as epinephrine and antiarrhythmic agents (amiodarone hydrochloride). IV fluid administration of $0.9 \%$ saline was continued throughout the experiment. Pigs were intubated, and anesthesia was maintained using an inhalation anesthetic of sevoflurane $(1 \%)$ in oxygen $(100 \%)$. The pigs were mechanically ventilated. Tramadol $\mathrm{HCl}\left(5 \mathrm{mg} / \mathrm{kg}\right.$, Trodon ${ }^{\circledR}$ Aju Pharm, South Korea) was administered IV pre- and postoperatively to reduce pain. The stent was inserted into the coronary artery of an 8-week-old pig, and its placement was confirmed using angiography. We used the left anterior descending (LAD) and left circumflex (LCX) arteries. The pigs were premedicated with $100 \mathrm{mg}$ of aspirin and $75 \mathrm{mg}$ of clopidogrel daily for 5 days prior to the procedure. The pigs were also treated with the immunosuppressant cyclosporine (CIPOL.N, Chong Kun Dang, South Korea) for 3 days after stent transplantation.

\section{Stenosis imaging: angiography, OCT, and $\mathrm{mCT}$}

Imaging analyses for stenosis evaluation were performed as previously described ${ }^{24}$. Briefly, a follow-up coronary angiogram was performed 4 weeks post-stent transplantation. Pigs were anesthetized and sacrificed using an overdose of potassium chloride at the end of the experiment. Hearts were rapidly removed, extracted and grossly sectioned at $1-\mathrm{cm}$ intervals. Myocardial sections were stained with a 2,3,5-triphenyl tetrazolium chloride (TTC) solution (1\% in phosphate-buffered saline) for $30 \mathrm{~min}$ at $37^{\circ} \mathrm{C}$. Sectioned heart tissues were fixed in $10 \%$ neutral buffered formalin overnight and embedded in paraffin for histological analyses.

\section{Angiography}

A 7F coronary artery-guiding catheter was placed within the opening of the coronary artery, and a baseline coronary angiogram was obtained using the nonionic contrast agent Omnihexol (Omnihexol 300, Korea United Pharm Co., Seoul, Korea) under fluoroscopic guidance and a mobile fluoroscopy system (BV Pulsera, Philips Medical Systems, Andover, MA, USA). Angiography was 
performed to confirm an obstruction of the mid-LAD. The guide wire, balloon catheter, and guiding catheter were removed, and the left carotid artery was ligated.

\section{Optical coherence tomography (OCT)}

The carotid artery was excised, and the neointima of pig blood vessels was measured using OCT (Model C7Xr OCT Imaging System). A guide wire was connected to a water box dedicated to in vitro experiments, and the coronary artery was fixed to the guide wire. An imaging catheter (C7 Dragonfly) was inserted through the guide wire into the coronary artery. OCT images were obtained via connecting the imaging catheter and the Dragonfly Duo, and neointimal vessels were measured using LightLab imaging (offline review workstation). We calculated the neointimal area by subtracting the lumen area from stent area (Neointimal area $=$ stent area - lumen area). Supplementary Table 2 presents the detailed analysis data.

\section{Microcomputed tomography (mCT)}

Tomograms of each sample were acquired using a microcomputed tomography scanner (SkyScan 1172, MA, Bruker). Harvested coronary artery tissues were stored in a $10 \%$ formalin solution and transferred to deionized water prior to $\mathrm{mCT}$ analysis. Tissue was fixed vertically in a cylindrical plastic container and mounted on a specimen stub using soft clay. The plastic container was sealed using paraffin film to prevent sample drying. Scanning was operated at $10-\mathrm{W}(100 \mathrm{kV} / 100 \mu \mathrm{A})$ X-ray generation power using an aluminum-copper filter. Images were recorded at a $0.4^{\circ}$ rotation for one step. Acquired images were reconstructed and visualized using software (NRecon/CTan, MA, Bruker). The in-stent restenosis area (ISR area) was calculated by subtracting the area of the lumen from the area within the stent strut.

\section{Immunohistochemical (IHC) staining}

The tissues were harvested, fixed in $4 \%$ paraformaldehyde (Wako), embedded in paraffin, and cut into 5- $\mu \mathrm{m}$ thick sections (Leica, Buffalo Grove, IL, USA). Immunohistochemistry was performed using anti-von Willebrand factor (vWF) (ab6994, Abcam, USA), anti-fibrin (Abcam, USA), anti-CD31 (ab108595, Abcam, USA) and antilamin A $+\mathrm{C}$ (Abcam, USA) antibodies at a 1:100 dilution and the appropriate secondary antibodies. Tissue samples were also stained with hematoxylin and eosin (H\&E) and Masson's trichrome. TTC, H\&E, and Masson's trichrome stains were performed to evaluate the infarcted area of the ventricle. An experienced cardiac pathologist performed histological evaluations of the infarcted myocardium.

\section{Tumor formation}

BALB/c nude mice (Orient Bio, South Korea) were used to evaluate tumor formation in vivo. U-Ms and HGF- or
VEGF-secreting U-Ms $\left(5 \times 10^{6}\right)$ were injected into mice in $50 \mu \mathrm{l}$ of serum-free DMEM and $50 \mu \mathrm{l}$ of Matrigel (Corning, NY, USA). MDA-MB-231 breast cancer cells were used as a positive control. Mice were observed for 4 weeks, and tumor volume was measured weekly. Mice were sacrificed using $\mathrm{CO}_{2}$, and tumor formation and weights were analyzed.

\section{Statistical analysis}

The data are expressed as the mean values \pm standard errors. The data were analyzed using Prism, and a $t$-test or ANOVA was used to compare the data of different experimental groups. Differences were considered significant when the $p$ values were $<0.05$.

\section{Results}

\section{Retention of HGF/U-Ms and VEGF/U-Ms growth and markers on stent material}

The full experiment was performed as described in the schematic image (Fig. 1a). We examined whether the engineered cells were successfully implanted on the stent. We confirmed that the stent material did not affect cell growth or the expression of the UCB-MSC markers. Characteristics of the VEGF/U-Ms and HGF/UMs were evaluated after long-term interactions with the stent material. Cells were seeded on the materials for more than 7 days, and doxycycline (Dox) increased HGF- and VEGF-secreting U-Ms proliferation (Fig. 1b-d). HGF and VEGF secretion from the Tet-on system was confirmed using immunoblotting (Fig. 1e). HGF and VEGF secretion did not alter MSC markers (positive CD116, CD105, and CD90 markers and negative CD45 and CD14 markers) (Fig. If \& sFig. 1A). We confirmed HGF and VEGF secretion and integration into the genome each time the cells were established on a stent (sFig. 1B). The effect of Dox on cell proliferation was a concern, but Dox did not affect MSC proliferation or morphology in this test (sFig. 2A \& 2B). We confirmed that U-Ms maintained their properties and growth on the stent, and we demonstrated that HGF and VEGF secretion from the engineered stem cells promoted cell proliferation.

\section{Cells remained on the stents after coronary stent transplantation}

Cell adhesion to the bare stent material is limited; therefore, we designed stents with biocompatible matrices. Polydopamine and fibronectin were sequentially conjugated onto a $\mathrm{CoCr}$ stent. Fibroblasts were seeded onto stent surfaces and subsequently decellularized to provide the cell-secreted extracellular matrices (Fig. 1a). U-Ms with eGFP genes integrated in the Chr 19 safeharbor site using TALEN were attached to the stent surfaces via the extracellular matrix. Cell adhesion was 
A
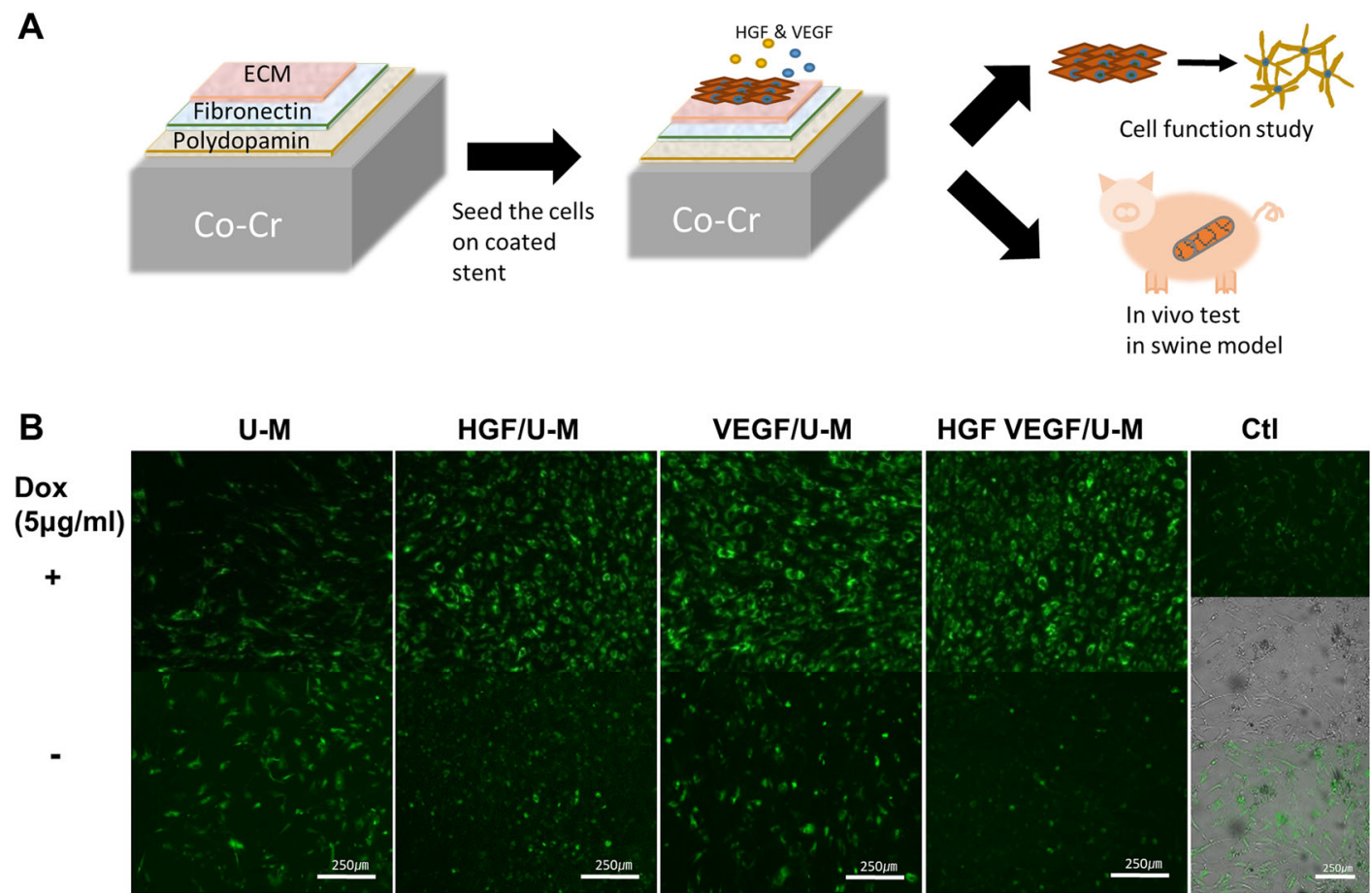

C

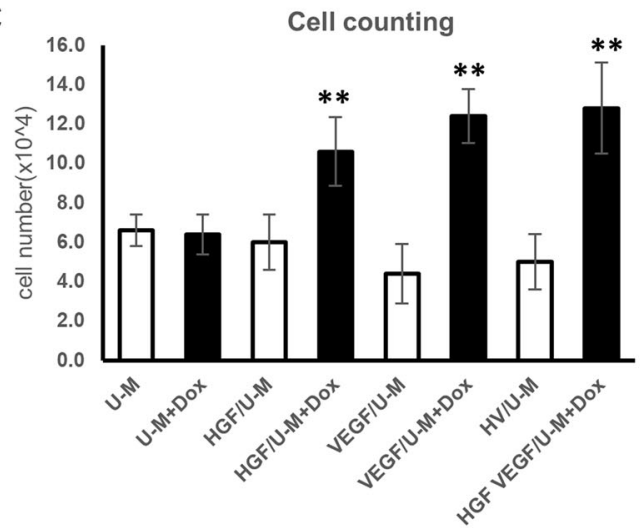

E

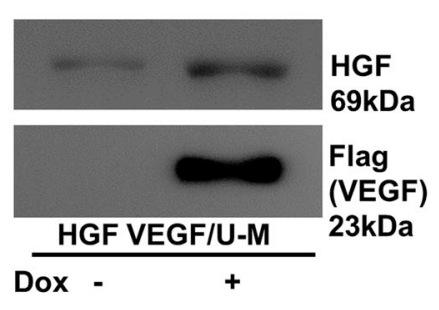

D $2.5 \quad$ Cell viability with Crystal Violet

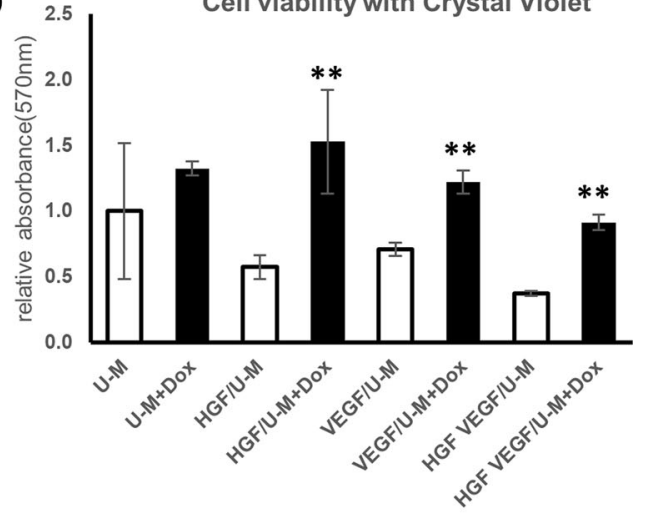

F

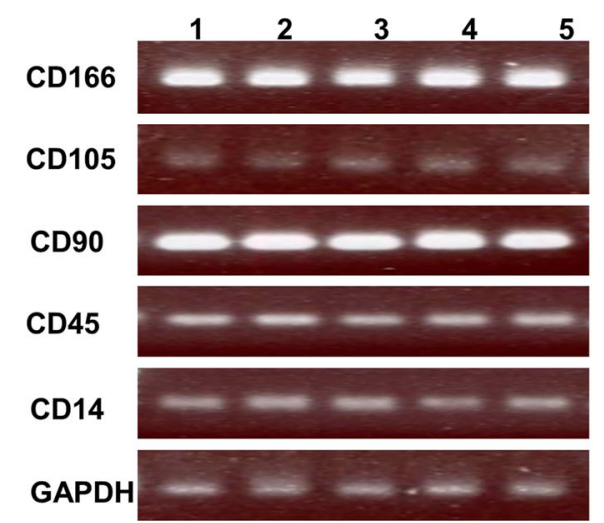

Fig. 1 (See legend on next page.) 
(see figure on previous page)

Fig. 1 The properties of HGF/U-Ms and VEGF/U-Ms are maintained on stent material. a Schematic illustration of the experiment using the stent with HGF/U-Ms and VEGF/U-Ms to secrete the angiogenic factors in an inducible manner. The stents were coated with polydopamine, fibronectin, and extracellular matrix (ECM), followed by in vitro and in vivo swine experiments. b Human U-Ms secreting HGF and VEGF were seeded onto the pre-coated material sheets to confirm cell viability on the stent material. The cells stained with green fluorescent dye were detected using

fluorescence microscopy. U-M human UCB-MSCs, Ct/ control. $N=3$. c The cells were counted at 7 days post seeding after detachment from the stent material and staining of dying cells using trypan blue ${ }^{* *}$ denotes a $p$-value $\left.<0.01\right)$. $\mathbf{d}$ Cell viability was analyzed using a crystal violet assay on the stent 7 days post seeding (** denotes a $p$-value $<0.01$ ). d, e HGF and VEGF secretion were detected in conditioned media using western blotting. HGF/UMs and VEGF/U-Ms were treated with $5 \mu \mathrm{g}$ of doxycycline for two days in a six-well plate. $\mathbf{f}$ MSC markers on the stent material were not altered, even in the HGF- and VEGF-secreting cells. Lane 1: U-Ms, 2: U-Ms + Dox, 3: U-Ms + Dox on material, 4: HGF/U-Ms + Dox on material, 5: HGF + VEGF/U-Ms + Dox on material. $N=3$ experiments per group

confirmed using fluorescence microscopy (Fig. 2a). Scanning electron microscopy (SEM) was performed to identify whether cells remained after mimicking stent implantation in the swine model due to concern about cell adherence on the stents after coronary artery transplantation. The surgical procedures led to some cell detachment due to the strong physical friction of balloon dilatation. However, cells remained on the lateral side of the stent after transplantation (Fig. 2b). These results suggest that the stem cells implanted on the stent remained after surgery.

\section{The effect of HGF and VEGF application periods on coronary restenosis}

Stents were applied to a swine model and observed for 2 weeks after stent implantation to evaluate the effects of the HGF/U-Ms- and VEGF/U-Ms-seeded stents on the coronary artery. The HGF/U-M group exhibited the lowest level of neointima formation among the bare metal stent (BMS) and stents with U-Ms, HGF/U-Ms, and VEGF/U-Ms (Fig. 3, HGF group). OCT and mCT analyses revealed that the HGF/U-M group exhibited the lowest amount of stenosis formation and a uniform pattern in the stenosis area (Fig. 3c). An even inner surface is a crucial/essential criterion for stent re-endothelialization. In contrast, excessive neointima formation was observed in the VEGF/U-M group after 2 weeks (Fig. 3, VEGF group). The VEGF group exhibited the lowest level of neointima formation in a 3-day trial (Supplemental Fig. 3), but this result was not observed at 2 weeks. The excessive amount of VEGF secreted from the VEGF/U-Ms may stimulate vascular smooth muscles during longer-term exposure to the cells. Other studies demonstrated that patients with increased VEGF after implantation exhibit a restenosis rate of $26.2 \%$ compared to that of $2.4 \%$ in patients with basal VEGF levels ${ }^{25,26}$. Restenosis was reduced in the HGF/U-M group, but this effect was not significant over the longer term. Therefore, the application of the cell population and the periodic conditioning of the HGF/U-Ms and VEGF/U-Ms must be optimized to promote re-endothelialization and minimize the neointima side effect.
Optimization of HGF- and VEGF-secreting stem cells and enhanced tube formation with the HGF/U-Ms and VEGF/UMs

Stents with VEGF-secreting stem cells and longer implant times ( 2 weeks) produced more severe restenosis (Fig. 3). This effect may have occurred because VEGF is one of the strongest factors that stimulate the fibrosis and proliferation of all cells in the vascular area ${ }^{26,27}$. However, cells that only secreted HGF or VEGF did not efficiently reduce restenosis (Fig. 3). The combination of VEGF-A and HGF promoted neovascularization, especially reendothelialization, under angiogenic conditions via enhanced intracellular signaling, which allows a more finely regulated control of the signaling molecules involved in the regulation of the cytoskeleton and cellular migration and morphogenesis ${ }^{28}$. Therefore, the combined condition is necessary, and the dose of HGF- and VEGFsecreting cells must be optimized. The amount of HGF and VEGF secreted from this system was analyzed in our previous studies. Fifty nanograms of VEGF and $2.2 \mathrm{ng}$ of HGF were secreted from $2 \times 10^{6}$ cells per day ${ }^{13,16}$. We maximized the HGF and minimized the VEGF based on these results because HGF promoted endothelial cells (ECs) more specifically, and VEGF increased overall vascular cell activity $^{29}$. A 5:1 ratio of HGF- to VEGFsecreting cells was investigated in vitro, and the cell proliferation was similar to the control single condition (Fig. 1, HGF VEGF/U-M group). This condition included a range that is suitable in vivo ${ }^{30}$.

A source of endothelial cells is needed to facilitate coronary in-stent re-endothelization. Endothelial progenitor cells (EPCs), embryonic stem cells (ESCs) and induced pluripotent stem cells (iPSCs) are used as endothelial cell sources in vitro. Notably, MSCs differentiate into endothelial cells ${ }^{31,32}$, and we presumed that our functional HGF VEGF/U-Ms would differentiate into ECs. Tube formation using HGF + VEGF/U-Ms was observed on Matrigel, even under the culture condition that excluded endothelial differentiation medium and bFGF. However, the U-Ms did not form the tube properly in the absence of Matrigel (Fig. 4). HGF + VEGF secretion from cells in response to doxycycline induction (HGF + 


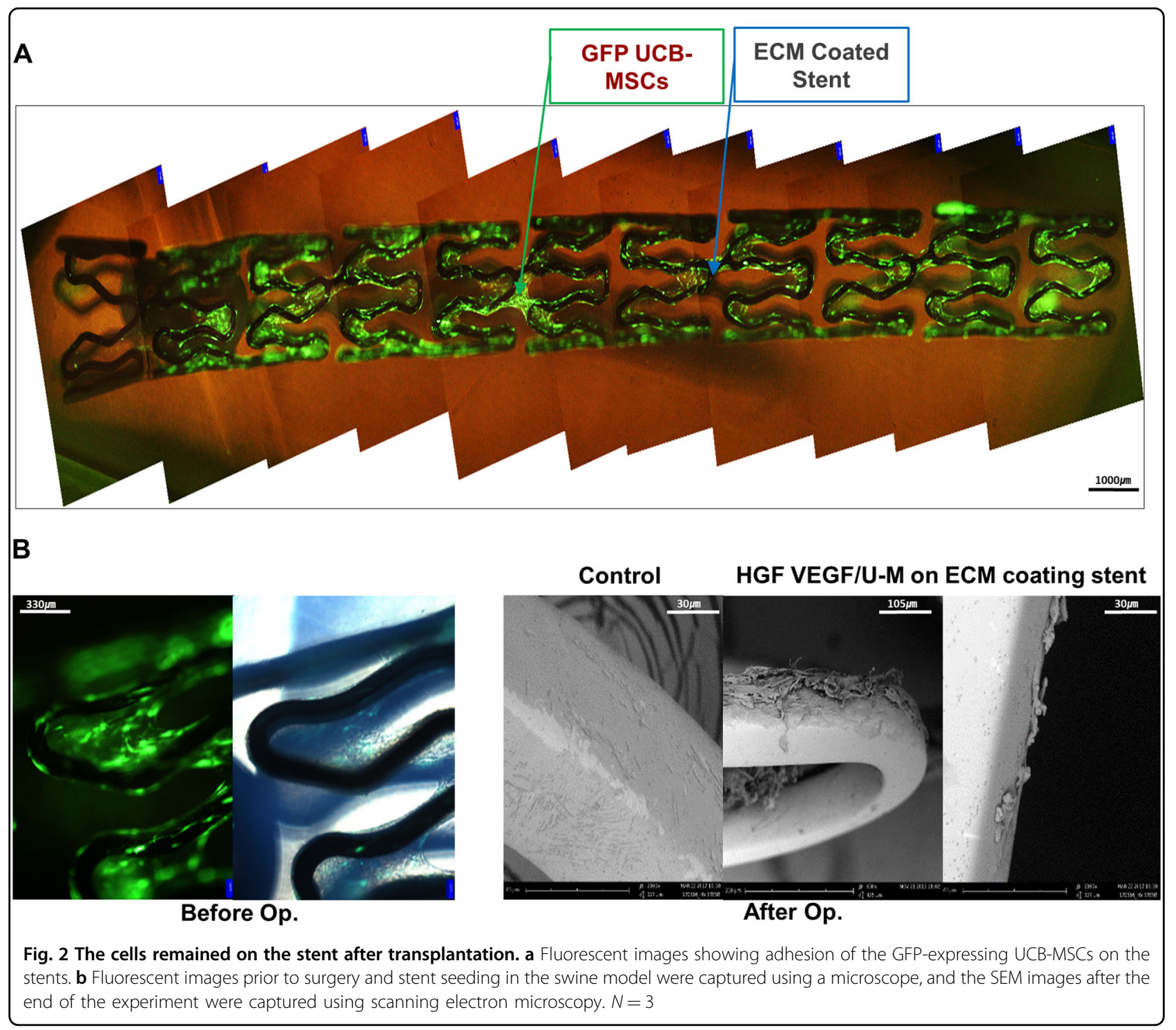

VEGF/U-Ms + Dox) enhanced the tube and branch numbers (Fig. 4b, c). The tubes persisted longer in the HGF + VEGF/U-Ms + Dox group (Fig. 4a). The 5:1 combination of HGF:VEGF/U-Ms in response to Dox stimulation resulted in better tube formation and branch numbers at early time points (3-9h) (Fig. 4 b, c). We examined HGF + VEGF-secreting stem cells at a 5:1 ratio in vivo for vascular re-endothelialization based on previous results and the tube formation results.

\section{Reduction of restenosis in the presence of HGF and VEGF} (5:1)

The HGF + VEGF/U-M combination stent was transplanted into the swine model to examine the efficacy of stents coated with HGF + VEGF (5:1)-secreting stem cells. Swine coronary arteries were observed 4 weeks after transplantation. The HGF/U-M + VEGF/U-M (+Dox) group exhibited the lowest restenosis compared to the BMS and U-M groups (Fig. 5). The HGF/U-M + VEGF/ U-M group exhibited the lowest neointimal area in OCT (Fig. 5a, b) and $\mathrm{mCT}$ analyses (Fig. 5c). Fibrin staining also revealed the lowest fibrosis in the HGF/U-M + VEGF/U$\mathrm{M}$ (+Dox) group (Fig. 5d, e). Live angiography analysis demonstrated normal blood flow in the HGF/U-M + VEGF/U-M (+Dox) group (Fig. 5f). Taken together, these results indicated that the swine coronary stents with HGF/U-Ms + VEGF/U-Ms in a 5:1 combination significantly decreased neointima formation and restenosis and provided the best blood flow.

\section{Enhanced re-endothelialization in stents with HGF/U-Ms + VEGF/U-Ms}

We stained the stent area of the coronary artery to visualize re-endothelialization after stent transplantation. 


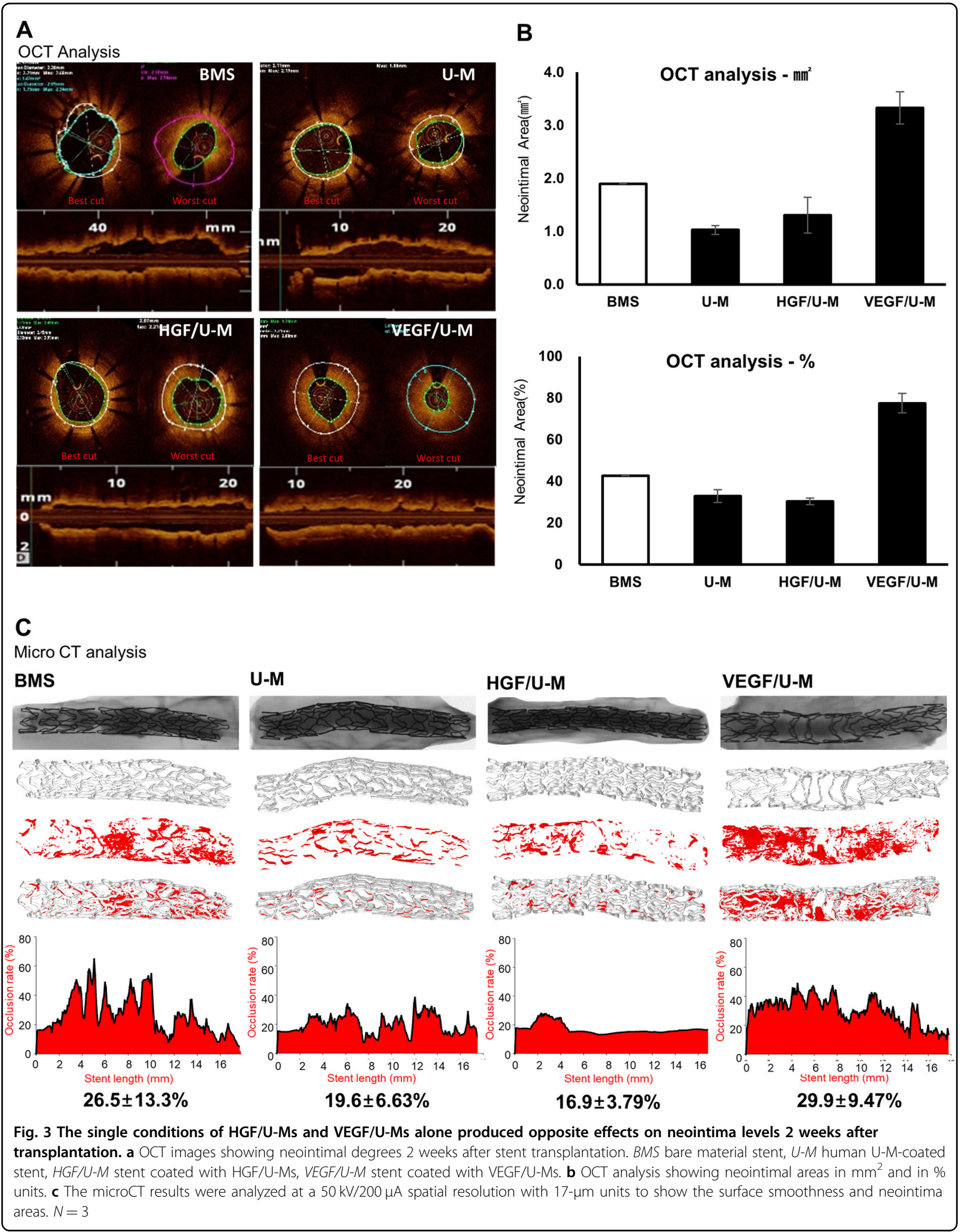




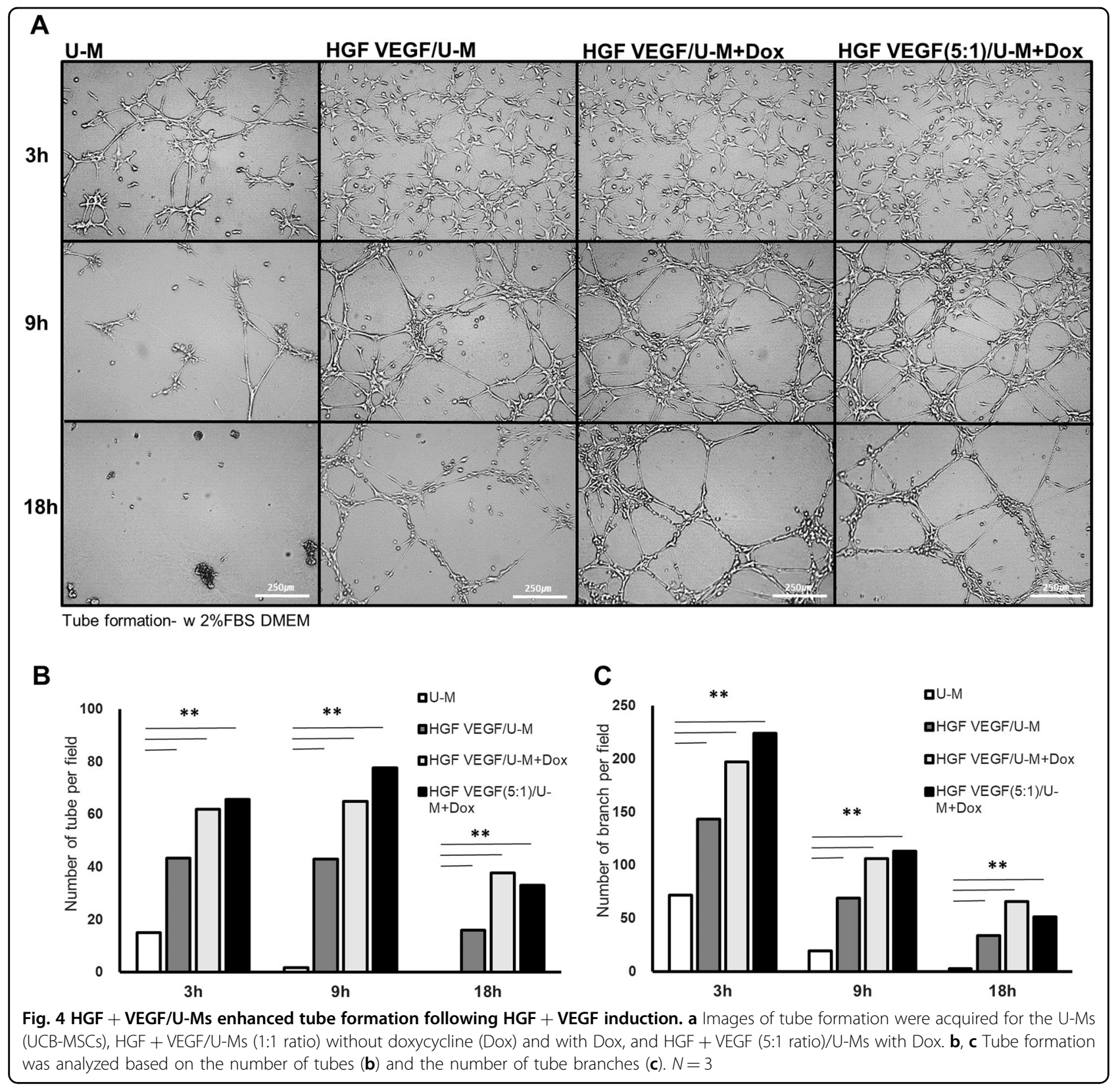

Immunostaining for the endothelial cell markers vWF and CD31 revealed EC layers in the HGF/U-M and VEGF/U-M groups 2 weeks after transplantation (Fig. 6a). The HGF/U-M + VEGF/U-M (5:1) group exhibited vWF and CD31 staining 4 weeks after transplantation, which indicates re-endothelialization. No vWF and CD31 staining was observed in the MSC only or BMS groups (Fig. 6c, d). Transplanted human MSCs were observed in the HGF/U-M and VEGF/U-M groups using genomic PCR to detect human-specific Alu (Fig. 6b). Human-specific Alu was detected in the HGF/U-M and VEGF/U-M stent groups, but not in the BMS group. These results suggest that human cells coated the endothelium after HGF/U-M or VEGF/U-M transplantation in the stent.

\section{HGF/U-Ms+VEGF/U-Ms did not induce tumor formation}

We performed a tumor formation assay in vivo to confirm the safety of the functional stem cells. The UCB stem cells that we used secreted VEGF and HGF growth factors; therefore, we hypothesized that these cells may generate cancer cells. Moreover, MSCs exhibit dual potential that can be either pro- or antitumorigenic potential ${ }^{33}$. The transplantation of 


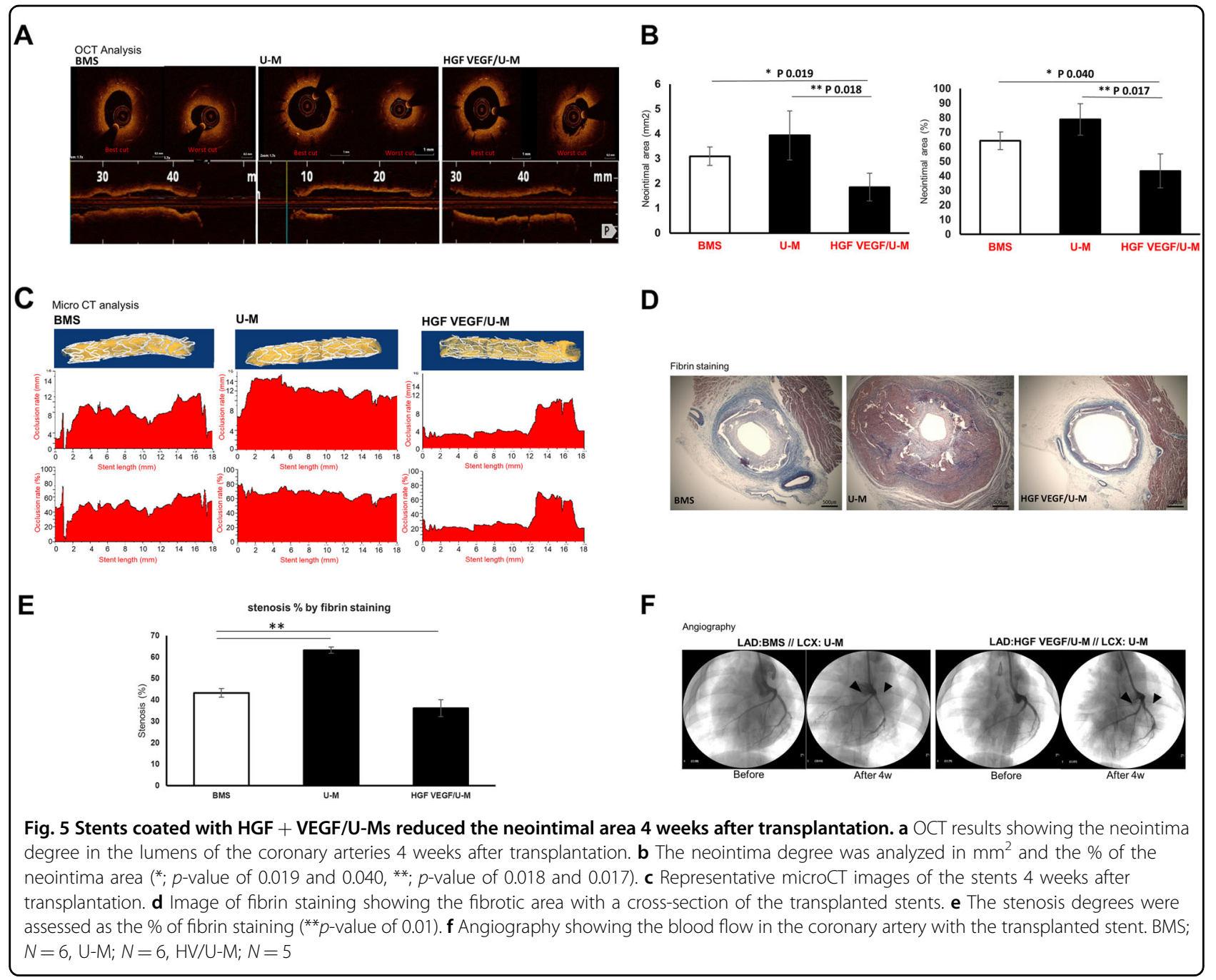

HGF/U-Ms + VEGF/U-Ms and U-M cells into nude mice produced no tumors up to 30 days post transplantation. However, the MDA-MB-231 breast cancer cell line formed a tumor mass (Fig. 7a, c). The size of the mixture of the HGF/U-Ms + VEGF/U-Ms and Matrigel decreased drastically and disappeared 1-2 weeks post transplantation (Fig. 7b).

\section{Discussion}

Growth factor-secreting stem cells play a pivotal role in stimulating re-endothelialization. These cells serve as carriers of growth factors and sources of endothelial cells. These carrier cells naturally provide HGF and VEGF to other cells, in a similar manner to that of endothelial cells. These cells also release HGF and VEGF in response to doxycycline in a controlled manner. This inducible system reduces the side effects of growth factor overproduction and helps identify optimal conditions for stent-mediated re-endothelialization.
The present study used HGF/U-Ms and VEGF/U-Ms on coronary stents to prevent restenosis and for therapeutic purposes. We also used these HGF- or VEGF-releasing functional stem cells as a source of endothelial cells on the stent for re-endothelialization. MSCs exhibit a high potential to become endothelial cells ${ }^{31,32}$. The present study demonstrated that HGF- or VEGF-releasing MSCs differentiated into endothelial cells and induced tube formation without additional growth factors (Fig. 4a). Angiogenesis and re-endothelialization are distinct processes, but these phenomena share the same regulatory molecular mechanism $^{33,34}$. The improved tube formation confirmed the reendothelialization of MSCs in vitro. We also demonstrated that the cells transdifferentiated into an endothelial cell layer, or at least provided a suitable microenvironment to form endothelial cells, in the swine model (Fig. 6).

However, some hurdles must be overcome in these cell-based stent systems. Cell attachment to the stent must be improved. A significant number of cells detached 


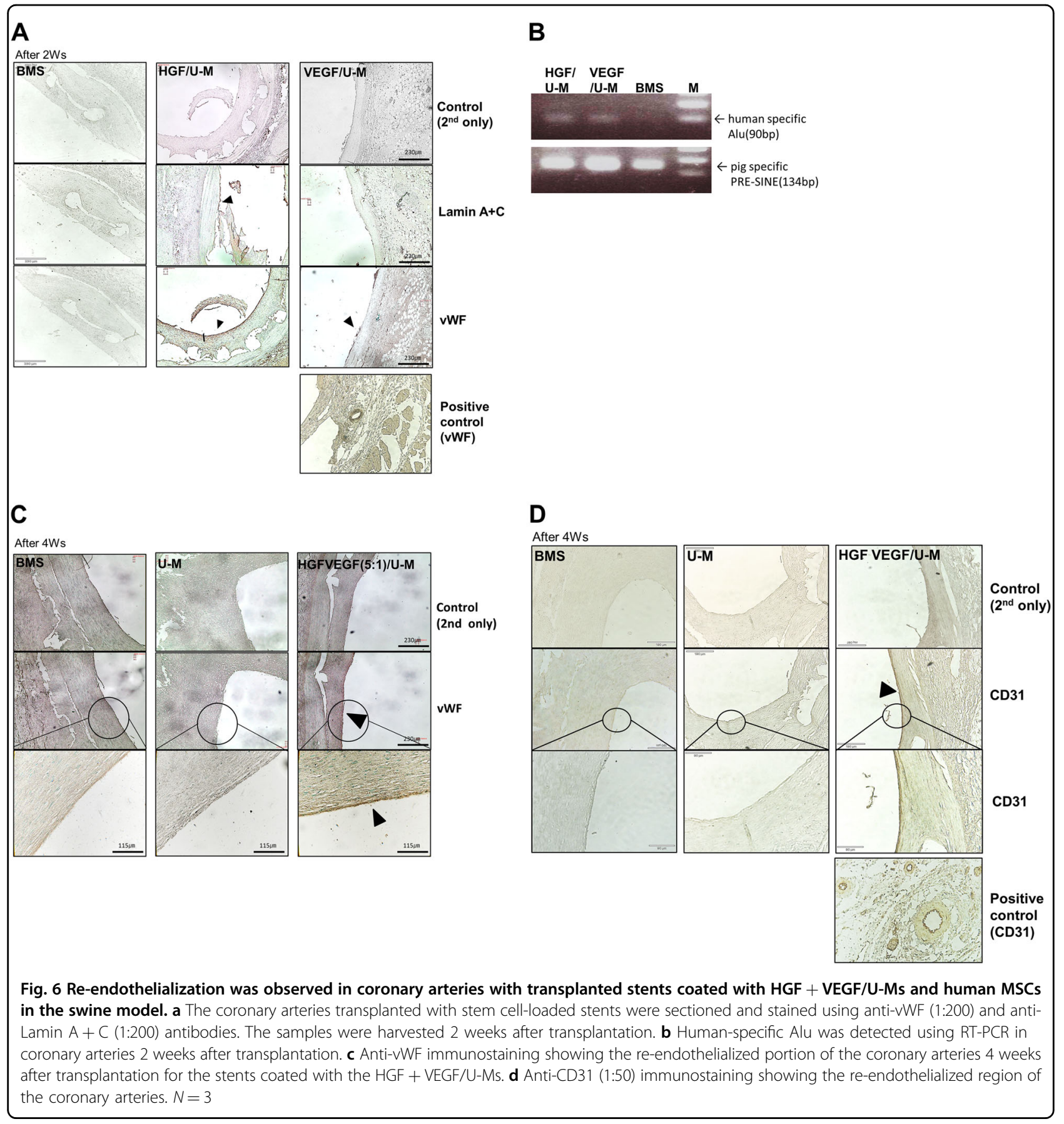

from the stent in our experiments due to the physical force exerted on the inner and outer surfaces of the stent during transplantation. Cells remained on the lateral side of the stent, but approximately only one third of the cells remained (Fig. 2b). More cells would enable greater restenosis reduction and re-endothelialization. Future studies should implement better cell attachment technology, and studies to improve cell attachment on the stents are ongoing ${ }^{35,36}$.
In this study, we found the optimal combination of HGF- and VEGF-secreting MSCs for the coronary stent to accomplish optimal restenosis reduction and endothelialization. We tried several different combinations of these two functional MSCs and found that the best combination of HGF- and VEGF-releasing cells was a 5:1 ratio. VEGF produced a significant effect on neointima reduction in the short-term trials ( 3 days) but not over periods of 2 weeks or longer (Fig. 3). VEGF promotes 


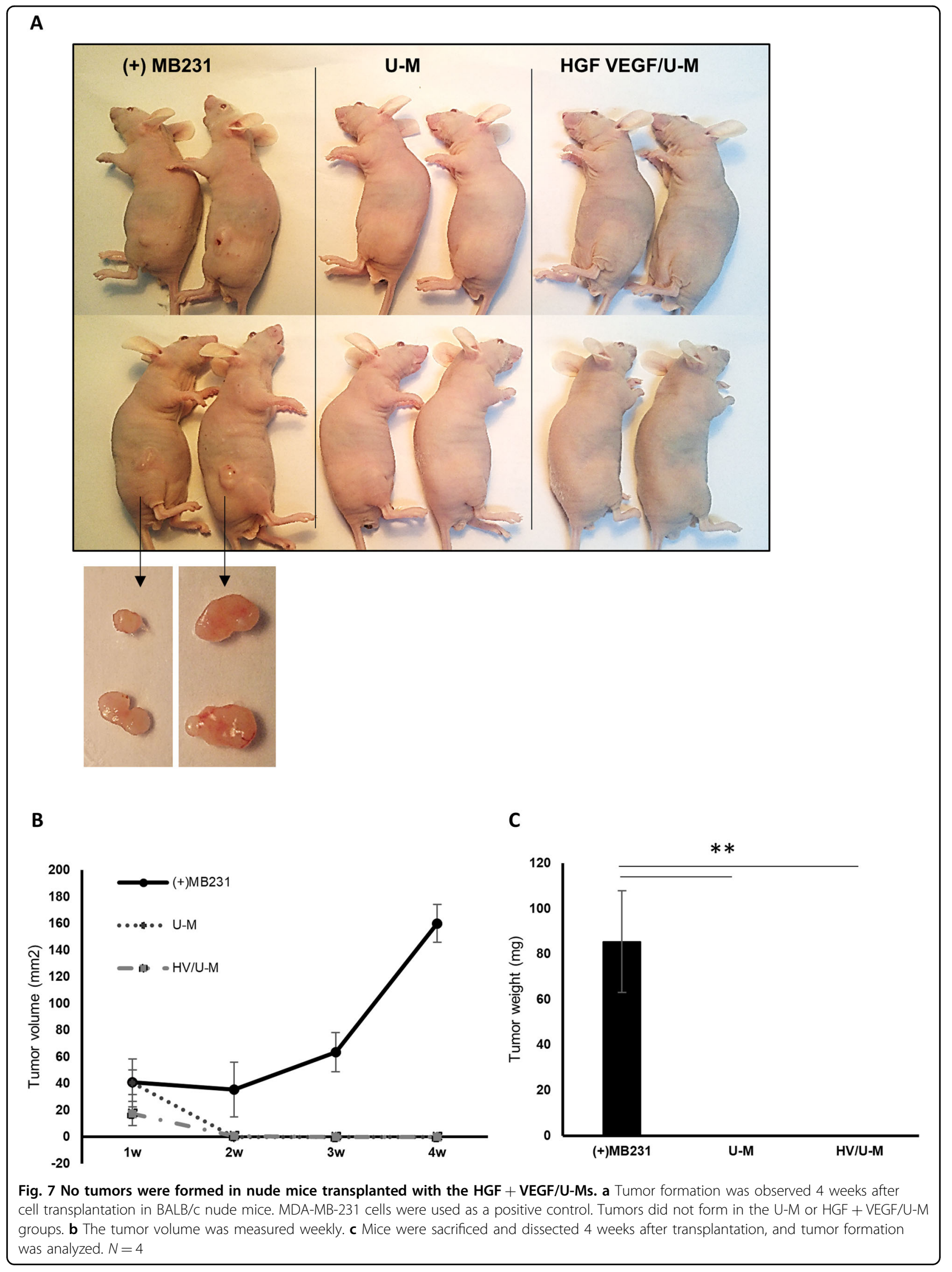


fibrosis in endothelial cells ${ }^{16,26,27}$. Therefore, longer exposure to a higher amount of VEGF may stimulate fibrosis instead of endothelialization. Conversely, HGF decreased restenosis in the 2-week trials, but this decrease was not sufficient compared to that of the BMS group. The seeding of HGF-secreting MSCs alone on the stent did not reduce restenosis 4 weeks after implantation. Several reports indicated that HGF exclusively stimulated endothelial cell growth and reduced fibrosis without replication of vascular smooth muscle cells ${ }^{14,29,37-39}$. However, the HGF/U-Ms did not survive longer than 2 weeks in vivo, and subsidiary support was necessary. We combined HGF-secreting cells with VEGF-secreting cells to solve this problem. The cross-talk between HGF and VEGF improves stem cell survival and the angioarchitecture and increases cell proliferation and migration ${ }^{28,34}$. This study found the optimal combination of HGF- and VEGF-secreting cells to promote re-endothelialization and minimize the restenosis side effects.

Drug-eluting stents may produce a dramatic reduction in stenosis, and several commercial drug-eluting stents are used clinically ${ }^{40,41}$. However, these stents exhibit the fatal problem of arterial restenosis at later stages. This problem partially occurs due to the inability to form a barrier and protect the artery. Therefore, reendothelialization is important to protect the coronary artery from restenosis ${ }^{42}$. Another drawback of drugeluting stents is that the drug itself is antiproliferative and exerts nonspecific effects on the surrounding cells. Therefore, the drug prevents the regeneration of VSMCs and ECs. No other method is available to promote EC regeneration using drug-eluting stents without affecting VSMCs. The functional HGF/VEGF-secreting stem cells seeded on the stent enhanced EC regeneration and prevented VSMC growth.

In summary, coating stents with HGF- and VEGFsecreting mesenchymal stem cells reduced the side effects of coronary stents via the promotion of reendothelialization. HGF promoted natural endothelialization and produced an even lumen side of the vessel wall. VEGF promoted fibrosis, but it also activated cell survival via the controlled release of the appropriate dose at the appropriate time, which promoted angiogenesis. Our strategy of using growth factor-secreting MSCs has significant implications for clinical stent therapy.

\section{Acknowledgements}

This research investigation was supported by grants from the National Research Foundation (NRF) funded by the Ministry of Science and ICT (grants nos. 2012M3A9C6049716 and 2016M3A9B6026771).

\footnotetext{
Author details

'Department of Biochemistry, BK21 PLUS Program for Creative Veterinary Science Research and Research Institute for Veterinary Science, College of Veterinary Medicine, Seoul National University, Seoul, South Korea.

${ }^{2}$ Department of Biomedical Laboratory Science, College of Medical Science,
}

Konyang University, Daejeon, South Korea. ${ }^{3}$ Center for Biomaterials, Korea Institute of Science and Technology, Seoul, South Korea. ${ }^{4}$ Department of Biomedical Science, CHA University, Sungnam, South Korea. ${ }^{5}$ Chonnam National University Hospital, Gwangju, South Korea. ${ }^{6}$ Present address: Futuristic Animal Resource and Research Center, Korea Research Institute of Bioscience and Biotechnology, Ochang, Chungbuk, Korea

\section{Conflict of interest}

The authors declare that they have no conflict of interest.

\section{Publisher's note}

Springer Nature remains neutral with regard to jurisdictional claims in published maps and institutional affiliations.

Supplementary information accompanies this paper at https://doi.org/ 10.1038/s12276-018-0143-9.

Received: 3 January 2018 Revised: 30 May 2018 Accepted: 7 June 2018. Published online: 3 September 2018

\section{References}

1. Faxon, D. P. et al. Atherosclerotic vascular disease conference: executive summary: atherosclerotic vascular disease conference proceeding for healthcare professionals from a special writing group of the American Heart Association. Circulation 109, 2595-2604 (2004).

2. GBD 2013 Mortality and Causes of Death Collaborators. Global, regional, and national age-sex specific all-cause and cause-specific mortality for 240 causes of death, 1990-2013: a systematic analysis for the Global Burden of Disease Study 2013. Lancet 385, 117-171 (2015).

3. Aziz, S. \& Ramsdale, D. R. Chronic total occlusions-a stiff challenge requiring a major breakthrough: is there light at the end of the tunnel. Heart $\mathbf{9 1}$ (Suppl 3), iii42-iii48 (2005).

4. Padfield, G. J., Newby, D. E. \& Mills, N. L. Understanding the role of endothelial progenitor cells in percutaneous coronary intervention. J. Am. Coll. Cardiol. 55, 1553-1565 (2010).

5. Reejhsinghani, R. \& Lotfi, A. S. Prevention of stent thrombosis: challenges and solutions. Vasc. Health Risk. Manag. 11, 93-106 (2015).

6. Li, T., Ding, Y., Wu, J., Shen, Y. \& Liu, X. [Advances in research on reendothelialization after intervention in artery]. Sheng Wu Yi Xue Gong. Cheng Xue Za Zhi 33, 177-183, 187 (2016).

7. Kleinedler, J. J., Foley, J. D., Orchard, E. A. \& Dugas, T. R. Novel nanocomposite stent coating releasing resveratrol and quercetin reduces neointimal hyperplasia and promotes re-endothelialization. J. Control Release 159, 27-33 (2012).

8. Versari, D., Lerman, L. O. \& Lerman, A. The importance of reendothelialization after arterial injury. Curr. Pharm. Des. 13, 1811-1824 (2007).

9. Tan, A., Alavijeh, M. S. \& Seifalian, A. M. Next generation stent coatings: convergence of biotechnology and nanotechnology. Trends Biotechnol. 30, 406-409 (2012)

10. Bedair, T. M., ElNaggar, M. A., Joung, Y. K. \& Han, D. K. Recent advances to accelerate re-endothelialization for vascular stents. J. Tissue Eng. 8, 2041731417731546 (2017).

11. Hoeben, A. et al. Vascular endothelial growth factor and angiogenesis. Pharmacol. Rev. 56, 549-580 (2004).

12. $\mathrm{Wu}, \mathrm{X}$. et al. Re-endothelialization study on endovascular stents seeded by endothelial cells through up- or downregulation of VEGF. ACS Appl. Mater. Interfaces 8, 7578-7589 (2016).

13. Chang, H. K. et al. Inducible HGF-secreting human umbilical cord bloodderived MSCs produced via TALEN-mediated genome editing promoted angiogenesis. Mol. Ther. 24, 1644-1654 (2016).

14. Ellison, G. M. et al. Endogenous cardiac stem cell activation by insulin-like growth factor-1/hepatocyte growth factor intracoronary injection fosters survival and regeneration of the infarcted pig heart. J. Am. Coll. Cardiol. 58, 977-986 (2011)

15. Huang, C., Zheng, X., Mei, H. \& Zhou, M. Rescuing impaired reendothelialization of drug-eluting stents using the hepatocyte growth factor. Ann. Vasc. Surg. 36, 273-282 (2016). 
16. Cho, H. M. et al. Targeted genome engineering to control VEGF expression in human umbilical cord blood-derived mesenchymal stem cells: potential implications for the treatment of myocardial infarction. Stem Cells Transl. Med. 6, 1040-1051 (2017)

17. Park, C. Study on Extracellular Matrix-coated Cardiovascular Materials for Encompassment of Outgrowth Endothelial Cells. MSc thesis, University of Science and Technology (2016).

18. Wozniak, M. A., Modzelewska, K., Kwong, L. \& Keely, P. J. Focal adhesion regulation of cell behavior. Biochim. Biophys. Acta 1692, 103-119 (2004).

19. Trappmann, B. et al. Extracellular-matrix tethering regulates stem-cell fate. Nat. Mater. 11, 642-649 (2012).

20. Kim, S. H., Turnbull, J. \& Guimond, S. Extracellular matrix and cell signalling: the dynamic cooperation of integrin, proteoglycan and growth factor receptor. J. Endocrinol. 209, 139-151 (2011).

21. Prewitz, M. C. et al. Tightly anchored tissue-mimetic matrices as instructive stem cell microenvironments. Nat. Methods 10, 788-794 (2013).

22. Waite, J. H. Surface chemistry: Mussel power. Nat. Mater. 7, 8-9 (2008).

23. Seo, Y. et al. Human umbilical cord blood-derived mesenchymal stem cells protect against neuronal cell death and ameliorate motor deficits in Niemann Pick type C1 mice. Cell Transplant. 20, 1033-1047 (2011).

24. Lim, K. S. et al. Effect of stents coated with a combination of sirolimus and alpha-lipoic acid in a porcine coronary restenosis model. J. Mater. Sci. Mater. Med. 27, 66 (2016).

25. Katsaros, K. M. et al. An increase of VEGF plasma levels is associated with restenosis of drug-eluting stents. Eurointervention 10, 224-230 (2014).

26. Yang, L. et al. Vascular endothelial growth factor promotes fibrosis resolution and repair in mice. Gastroenterology 146, 1339-1350.e1 (2014).

27. Park, S., Kim, J. W., Kim, J. H., Lim, C. W. \& Kim, B. Differential roles of angiogenesis in the induction of fibrogenesis and the resolution of fibrosis in liver. Biol. Pharm. Bull. 38, 980-985 (2015).

28. Sulpice, E. et al. Cross-talk between the VEGF-A and HGF signalling pathways in endothelial cells. Biol. Cell 101, 525-539 (2009).

29. Hayashi, K. et al. In vivo transfer of human hepatocyte growth factor gene accelerates re-endothelialization and inhibits neointimal formation after balloon injury in rat model. Gene Ther. 7, 1664-1671 (2000).
30. Deindl, E. \& Kupatt, C. Therapeutic Neovascularization-Quo Vadis (Springer Klinikum Grosshadern, Munich, 2007).

31. Oswald, J. et al. Mesenchymal stem cells can be differentiated into endothelial cells in vitro. Stem Cells 22, 377-384 (2004).

32. Janeczek Portalska, K. et al. Endothelial differentiation of mesenchymal stromal cells. PLOS ONE 7, e46842 (2012).

33. Bergfeld, S. A. \& DeClerck, Y. A. Bone marrow-derived mesenchymal stem cells and the tumor microenvironment. Cancer Metastas-. Rev. 29, 249-261 (2010).

34. Michael, S. C. Angiogenesis: from the molecular to integrative pharmacology. Proceedings of the 5th biannual meeting. Crete, Greece, July 1-7, 1999. Adv Exp. Med. Biol. 476, 1-382 (2000)

35. Zhou, Z. et al. Development of transgenic endothelial progenitor cell-seeded stents. J. Biomed. Mater. Res. A 91, 623-628 (2009).

36. Raina, T. et al. Coronary stents seeded with human trophoblastic endovascular progenitor cells show accelerated strut coverage without excessive neointimal proliferation in a porcine model. Eurointervention 10, 709-716 (2014).

37. Perin, E. C. et al. Human hepatocyte growth factor (VM202) gene therapy via transendocardial injection in a pig model of chronic myocardial ischemia. J. Card. Fail. 17, 601-611 (2011).

38. Van Belle, E. et al. Potentiated angiogenic effect of scatter factor/hepatocyte growth factor via induction of vascular endothelial growth factor: the case for paracrine amplification of angiogenesis. Circulation 97, 381-390 (1998).

39. Xia, J. L., Dai, C., Michalopoulos, G. K. \& Liu, Y. Hepatocyte growth factor attenuates liver fibrosis induced by bile duct ligation. Am. J. Pathol. 168, 1500-1512 (2006).

40. Htay, T. \& Liu, M. W. Drug-eluting stent: a review and update. Vasc. Health Risk. Manag. 1, 263-276 (2005).

41. Taniwaki, M. et al. 4-year clinical outcomes and predictors of repeat revascularization in patients treated with new-generation drug-eluting stents: a report from the RESOLUTE All-Comers trial (A Randomized Comparison of a Zotarolimus-Eluting Stent With an Everolimus-Eluting Stent for Percutaneous Coronary Intervention). J. Am. Coll. Cardiol. 63, 1617-1625 (2014).

42. Douglas, G. et al. Endothelial cell repopulation after stenting determines instent neointima formation: effects of bare-metal vs. drug-eluting stents and genetic endothelial cell modification. Eur. Heart J. 34, 3378-3388 (2013). 